\title{
Modified Stancu operators based on inverse Polya Eggenberger distribution
}

\section{Sheetal Deshwal' ${ }^{1}$ PN Agrawall ${ }^{1}$ and Serkan Araci ${ }^{2 *}$ (1)}

\section{${ }^{*}$ Correspondence:}

mtsrkn@hotmail.com

${ }^{2}$ Department of Economics, Faculty of Economics, Administrative and

Social Sciences, Hasan Kalyoncu University, Gaziantep, 27410, Turkey Full list of author information is available at the end of the article

\begin{abstract}
In this paper, we construct a sequence of modified Stancu-Baskakov operators for a real valued function bounded on $[0, \infty)$, based on a function $\tau(x)$. This function $\tau(x)$ is infinite times continuously differentiable on $[0, \infty)$ and satisfy the conditions $\tau(0)=0, \tau^{\prime}(x)>0$ and $\tau^{\prime \prime}(x)$ is bounded for all $x \in[0, \infty)$. We study the degree of approximation of these operators by means of the Peetre K-functional and the Ditzian-Totik modulus of smoothness. The quantitative Voronovskaja-type theorems are also established in terms of the first order Ditzian-Totik modulus of smoothness.
\end{abstract}

MSC: $41 \mathrm{~A} 36 ; 41 \mathrm{~A} 25$

Keywords: Baskakov operator; Ditzian-Totik modulus of smoothness; rate of convergence; Voronovskaja-type theorem

\section{Introduction}

In 1923, Eggenberger and Pólya [1] were the first to introduce Pólya-Eggenberger distribution. After that, in 1969, Johnson and Kotz [2] gave a short discussion of PólyaEggenberger distribution.

The Pólya-Eggenberger distribution $X[2]$ is defined by

$$
\begin{aligned}
\operatorname{Pr}(X=k)=\left(\begin{array}{l}
n \\
k
\end{array}\right) \frac{a(a+s) \cdots(a+(u-1) s) b(b+s) \cdots(b+(n-u-1) s)}{(a+b)(a+b+s) \cdots(a+b+(n-1) s)}, \\
\quad k=0,1,2, \ldots, n .
\end{aligned}
$$

The inverse Pólya-Eggenberger distribution $N$ is defined by

$$
\begin{aligned}
& \operatorname{Pr}(N=n+k)=\left(\begin{array}{c}
(n+k-1) \\
k
\end{array}\right) \frac{a(a+s) \cdots(a+(n-1) s) b(b+s) \cdots(b+(k-1) s)}{(a+b)(a+b+s) \cdots(a+b+(n+k-1) s)}, \\
& \quad k=0,1,2, \ldots, n .
\end{aligned}
$$

In 1970, Stancu [3] introduced a generalization of the Baskakov operators based on inverse Pólya-Eggenberger distribution for a real valued bounded function on $[0, \infty)$, defined by

$$
V_{n}^{[\alpha]}(f ; x)=\sum_{k=0}^{\infty} v_{n, k}(x, \alpha) f\left(\frac{k}{n}\right)=\sum_{k=0}^{\infty}\left(\begin{array}{c}
n+k-1 \\
k
\end{array}\right) \frac{1^{[n,-\alpha]} x^{[k,-\alpha]}}{(1+x)^{[n+k,-\alpha]}} f\left(\frac{k}{n}\right),
$$

(0) The Author(s) 2017. This article is distributed under the terms of the Creative Commons Attribution 4.0 International License (http://creativecommons.org/licenses/by/4.0/), which permits unrestricted use, distribution, and reproduction in any medium, provided you give appropriate credit to the original author(s) and the source, provide a link to the Creative Commons license, and indicate if changes were made. 
where $\alpha$ is a non-negative parameter which may depend only on $n \in \mathbb{N}$ and $a^{[n, h]}=a(a-$ $h)(a-2 h) \cdots(a-(n-1) h), a^{[0, h]}=1$ is known as a factorial power of $a$ with increment $h$. For $\alpha=0$, the operator (1.3) reduces to Baskakov operators [4].

In 1989, Razi [5] studied convergence properties of Stancu-Kantorovich operators based on Pólya-Eggenberger distribution. Very recently, Deo et al. [6] introduced a StancuKantorovich operators based on inverse Pólya-Eggenberger distribution and studied some of its convergence properties. For some other relevant research in this direction we refer the reader to [7-9].

Now, for $\alpha=\frac{1}{n}$, we get a special case of Stancu-Baskakov operators (1.3) defined as

$$
V_{n}^{\left\langle\frac{1}{n}\right\rangle}(f ; x)=\frac{(2 n-1) !}{(n-1) !} \sum_{k=0}^{\infty}\left(\begin{array}{c}
n+k-1 \\
k
\end{array}\right) \frac{(n x)_{k}}{(n+n x)_{n+k}} f\left(\frac{k}{n}\right)
$$

where $(a)_{n}:=a^{[n,-1]}=a(a+1) \cdots(a+(n-1))$ is called the Pochhammer symbol.

For the Lupas operator, given by

$$
L_{n}(f ; x)=\sum_{k=0}^{\infty}\left(\begin{array}{c}
n+k-1 \\
k
\end{array}\right) \frac{t^{k}}{(1+t)^{n+k}} f\left(\frac{k}{n}\right) \text {, }
$$

let $\mu_{n, m}(x)=L_{n}\left(t^{m} ; x\right), m \in \mathbb{N} \cup\{0\}$ be the $m$ th order moment.

Lemma 1 For the function $\mu_{n, m}(x)$, we have $\mu_{n, 0}(x)=1$ and we have the recurrence relation

$$
x(1+x) \mu_{n, m}^{\prime}(x)=n \mu_{n, m+1}(x)-n x \mu_{n, m}(x), \quad m \in \mathbb{N} \cup\{0\},
$$

where $\mu_{n, m}^{\prime}(x)$ is the derivative of $\mu_{n, m}(x)$.

Proof On differentiating $\mu_{n, m}(x)$ with respect to $x$, the proof of the recurrence relation easily follows; hence the details are omitted.

Remark 1 From Lemma 1, we have

$$
\mu_{n, 1}(x)=x, \quad \mu_{n, 2}(x)=\frac{x+(n+1) x^{2}}{n}, \quad \mu_{n, 3}(x)=\frac{(n+1)(n+2) x^{3}+3(n+1) x^{2}+x}{n^{2}} .
$$

The values of the Stancu-Baskakov operators (1.4) for the test functions $e_{i}(t)=t^{i}, i=$ $0,1,2$, are given in the following lemma.

Lemma 2 ([10]) The Stancu-Baskakov operators (1.4) verify:

(i) $V_{n}^{\left\langle\frac{1}{n}\right\rangle}(1 ; x)=1$,

(ii) $V_{n}^{\left\langle\frac{1}{n}\right\rangle}(t ; x)=\frac{n x}{n-1}$,

(iii) $V_{n}^{\left\langle\frac{1}{n}\right\rangle}\left(t^{2} ; x\right)=\frac{n^{2}}{(n-1)(n-2)}\left[x^{2}+\frac{x(x+1)}{n}+\frac{1}{n}\left(1-\frac{1}{n}\right) x\right]$.

(iv) $V_{n}^{\left\langle\frac{1}{n}\right\rangle}\left(t^{3} ; x\right)=\frac{n^{3}}{(n-1)(n-2)(n-3)}\left[\frac{(n+1)(n+2)}{n^{2}} x^{3}+\frac{3\left(2 n^{2}+n-1\right)}{n^{3}} x^{2}+\frac{(2 n-1)(3 n-1)}{n^{4}} x\right]$

(v) $V_{n}^{\left\langle\frac{1}{n}\right\rangle}\left(t^{4} ; x\right)=\frac{n^{4}}{(n-1)(n-2)(n-3)(n-4)}\left[\frac{(n+1)(n+2)(n+3)}{n^{3}} x^{4}+\frac{6(n+1)(n+2)(2 n-1)}{n^{4}} x^{3}+\frac{6\left(6 n^{3}+n^{2}-4 n+1\right)}{n^{5}} x^{2}+\right.$ $\left.\frac{26 n^{2}-27 n+7}{n^{5}} x\right]$. 
Proof The identities (i)-(iii) are proved in [10], hence we give the proof of the identity (iv). The identity (v) can be proved in a similar manner.

We have

$$
\begin{aligned}
V_{n}^{\left\langle\frac{1}{n}\right\rangle}\left(t^{3} ; x\right) & =\frac{(2 n-1 !)}{(n-1) !} \sum_{k=0}^{\infty}\left(\begin{array}{c}
(n+k-1) \\
k
\end{array}\right) \frac{(n x)_{k}}{(n+n x)_{n+k}}\left(\frac{k}{n}\right)^{3} \\
& =\frac{1}{B(n x, n)} \int_{0}^{\infty} \frac{t^{n x-1}}{(1+t)^{n x+n}} \mu_{n, 3}(t) d t,
\end{aligned}
$$

where $B(n x, n)$ is the Beta function.

Therefore using Remark 1 , we get

$$
V_{n}^{\left\langle\frac{1}{n}\right\rangle}\left(t^{3} ; x\right)=\frac{1}{B(n x, n)} \int_{0}^{\infty} \frac{t^{n x-1}}{(1+t)^{n x+n}}\left[\frac{(n+1)(n+2) t^{3}+3(n+1) t^{2}+t}{n^{2}}\right] d t .
$$

Now, by a simple calculation, we get the required result.

As a consequence of Lemma 2, we obtain the following.

Lemma 3 For the Stancu-Baskakov operator (1.4), the following equalities hold:

(i) $V_{n}^{\left\langle\frac{1}{n}\right\rangle}((t-x) ; x)=\frac{x}{n-1}$,

(ii) $V_{n}^{\left\langle\frac{1}{n}\right\rangle}\left((t-x)^{2} ; x\right)=\frac{2 n x(x+1)+(2 x-1) x}{(n-1)(n-2)}$,

(iii) $V_{n}^{\left\langle\frac{1}{n}\right\rangle}\left((t-x)^{4} ; x\right)=\frac{1}{n(n-1)(n-2)(n-3)(n-4)}\left[12 n\left(n^{2}-13 n+2\right) x^{3}(x+1)+12 n\left(n^{2}+8 n-\right.\right.$ 13) $\left.x^{2}(x+1)+\left(26 n^{2}+48 n-22\right) x(x+1)+(29-75 n) x\right]$.

Let $0 \leq r_{n}(x) \leq 1$ be a sequence of continuous functions for each $x \in[0,1]$ and $n \in \mathbb{N}$. Using this sequence $r_{n}(x)$, for any $f \in C[0,1]$, King [11] proposed the following modification of the Bernstein polynomial for a better approximation:

$$
\left(\left(B_{n} f\right) \circ r_{n}\right)(x)=\sum_{k=0}^{n} f\left(\frac{k}{n}\right)\left(\begin{array}{l}
n \\
k
\end{array}\right)\left(r_{n}(x)\right)^{k}\left(1-r_{n}(x)\right)^{n-k} .
$$

Gonska et al. [12] introduced a sequence of King-type operators $D_{n}^{\tau}: C[0,1] \rightarrow C[0,1]$ defined as

$$
D_{n}^{\tau} f=\left(B_{n} f\right) \circ\left(B_{n} \tau\right)^{-1} \circ \tau,
$$

where $\tau \in C[0,1]$ such that $\tau(0)=0, \tau(1)=1$ and $\tau^{\prime}(x)>0$ for each $x \in[0,1]$ and studied global smoothness preservation, the approximation of decreasing and convex functions, the validity of a Voronovskaja-type theorem and a recursion formula generalizing a corresponding result for the classical Bernstein operators.

Motivated by the above work, in the present paper we introduce modified StancuBaskakov operators based on a function $\tau(x)$ and obtain the rate of approximation of these operators with the help of Peetre's K-functional and the Ditzian-Totik modulus of smoothness. Also, we prove a quantitative Voronovskaja-type theorem by using the first order Ditzian-Totik modulus of smoothness.

Throughout this paper, we assume that $C$ denotes a constant not necessarily the same at each occurence. 


\section{Modified Stancu-Baskakov operators}

Let $\tau(x)$ be continuously differentiable $\infty$ times on $[0, \infty)$, such that $\tau(0)=0, \tau^{\prime}(x)>0$ and $\tau^{\prime \prime}(x)$ is bounded for all $x \in[0, \infty)$. We introduce a sequence of Stancu-Baskakov operators for $f \in C_{B}[0, \infty)$, the space of all continuous and bounded functions on $[0, \infty)$, endowed with the norm $\|f\|=\sup _{x \in[0, \infty)}|f(x)|$, by

$$
V_{n}^{\left\langle\frac{1}{n}, \tau\right\rangle}(f ; x)=\sum_{k=0}^{\infty} p_{n, k}^{\left\langle\frac{1}{n}, \tau\right\rangle}(x)\left(f \circ \tau^{-1}\right)\left(\frac{k}{n}\right), \quad x \in[0, \infty),
$$

where

$$
p_{n, k}^{\left\langle\frac{1}{n}, \tau\right\rangle}(x)=\frac{(2 n-1) !}{(n-1) !}\left(\begin{array}{c}
n+k-1 \\
k
\end{array}\right) \frac{(n \tau(x))_{k}}{(n(1+\tau(x)))_{n+k}} .
$$

Lemma 4 The operator defined by (2.1) satisfies the following equalities:

(i) $V_{n}^{\left\langle\frac{1}{n}, \tau\right\rangle}(1 ; x)=1$

(ii) $V_{n}^{\left\langle\frac{1}{n}, \tau\right\rangle}(\tau(t) ; x)=\frac{n \tau(x)}{n-1}$,

(iii) $V_{n}^{\left\langle\frac{1}{n}, \tau\right\rangle}\left(\tau^{2}(t) ; x\right)=\frac{n^{2}}{(n-1)(n-2)}\left[\tau^{2}(x)+\frac{\tau(x)(\tau(x)+1)}{n}+\frac{1}{n}\left(1-\frac{1}{n}\right) \tau(x)\right]$,

(iv) $V_{n}^{\left\langle\frac{1}{n}\right\rangle}\left(\tau^{3}(t) ; x\right)=\frac{n^{3}}{(n-1)(n-2)(n-3)}\left[\frac{(n+1)(n+2)}{n^{2}} \tau^{3}(x)+\frac{3\left(2 n^{2}+n-1\right)}{n^{3}} \tau^{2}(x)+\frac{(2 n-1)(3 n-1)}{n^{4}} \tau(x)\right]$,

(v) $V_{n}^{\left\langle\frac{1}{n}\right\rangle}\left(\tau^{4}(t) ; x\right)=\frac{n^{4}}{(n-1)(n-2)(n-3)(n-4)}\left[\frac{(n+1)(n+2)(n+3)}{n^{3}} \tau^{4}(x)+\frac{6(n+1)(n+2)(2 n-1)}{n^{4}} \tau^{3}(x)+\right.$ $\left.\frac{6\left(6 n^{3}+n^{2}-4 n+1\right)}{n^{5}} \tau^{2}(x)+\frac{26 n^{2}-27 n+7}{n^{5}} \tau(x)\right]$.

Proof The proof of lemma is straightforward on using Lemma 2. Hence we omit the details.

Let the $m$ th order central moment for the operators given by (2.1) be defined as

$$
\mu_{n, m}^{\tau}(x)=V_{n}^{\left\langle\frac{1}{n}, \tau\right\rangle}\left((\tau(t)-\tau(x))^{m} ; x\right)
$$

Lemma 5 For the central moment operator $\mu_{n, m}^{\tau}(x)$, the following equalities hold:

(i) $\mu_{n, 1}^{\tau}(x)=\frac{\tau(x)}{n-1}$

(ii) $\mu_{n, 2}^{\tau}(x)=\frac{2 n \phi_{\tau}^{2}(x)+(2 \tau(x)-1) \tau(x)}{(n-1)(n-2)}$,

(iii) $\mu_{n, 4}^{\tau}(x)=\frac{1}{n(n-1)(n-2)(n-3)(n-4)}\left[12 n\left(n^{2}-13 n+2\right) \tau^{2}(x) \phi_{\tau}^{2}(x)+12 n\left(n^{2}+8 n-\right.\right.$ 13) $\left.\tau(x) \phi_{\tau}^{2}(x)+\left(26 n^{2}+48 n-22\right) \phi_{\tau}^{2}(x)+(29-75 n) \tau(x)\right]$,

where $\phi_{\tau(x)}^{2}(x)=\tau(x)(\tau(x)+1)$.

Proof Using the definition (2.1) of the modified Stancu-Baskakov operators and Lemma 4, the proof of the lemma easily follows. Hence, the details are omitted.

Let

$$
W^{2}=\left\{g \in C_{B}[0, \infty): g^{\prime}, g^{\prime \prime} \in C_{B}[0, \infty)\right\}
$$

For $f \in C_{B}[0, \infty)$ and $\delta>0$, the Peetre $K$-functional [13] is defined by

$$
K(f ; \delta)=\inf _{g \in W^{2}}\left\{\|f-g\|+\delta\|g\|_{W^{2}}\right\}
$$


where

$$
\|g\|_{W^{2}}=\|g\|+\left\|g^{\prime}\right\|+\left\|g^{\prime \prime}\right\| .
$$

From [14], Proposition 3.4.1, there exists a constant $C>0$ independent of $f$ and $\delta$ such that

$$
K(f ; \delta) \leq C\left(\omega_{2}(f ; \sqrt{\delta})+\min \{1, \delta\}\|f\|\right),
$$

where $\omega_{2}$ is the second order modulus of smoothness of $f \in C_{B}[0, \infty)$ and is defined as

$$
\omega_{2}(f ; \delta)=\sup _{0<|h| \leq \delta} \sup _{x, x+2 h \in[0, \infty)}|f(x+2 h)-2 f(x+h)+f(x)| .
$$

In the following, we assume that $\inf _{x \in[0, \infty)} \tau^{\prime}(x) \geq a, a \in \mathbb{R}^{+}:=(0, \infty)$.

Next, we recall the definitions of the Ditzian-Totik first order modulus of smoothness and the $K$-functional [15]. Let $\phi_{\tau}(x):=\sqrt{\tau(x)(1+\tau(x))}$ and $f \in C_{B}[0, \infty)$. The first order modulus of smoothness is given by

$$
\omega_{\phi_{\tau}}(f ; t)=\sup _{0<h \leq t}\left\{\left|f\left(x+\frac{h \phi_{\tau}(x)}{2}\right)-f\left(x-\frac{h \phi_{\tau}(x)}{2}\right)\right|, x \pm \frac{h \phi_{\tau}(x)}{2} \in[0, \infty)\right\} .
$$

Further, the appropriate $K$-functional is defined by

$$
K_{\phi_{\tau}}(f ; t)=\inf _{g \in W_{\phi_{\tau}}[0, \infty)}\left\{\|f-g\|+t\left\|\phi_{\tau} g^{\prime}\right\|\right\} \quad(t>0),
$$

where $W_{\phi_{\tau}}[0, \infty)=\left\{g: g \in A C_{\mathrm{loc}}[0, \infty),\left\|\phi_{\tau} g^{\prime}\right\|<\infty\right\}$ and $g \in A C_{\mathrm{loc}}[0, \infty)$ means that $g$ is absolutely continuous on every interval $[a, b] \subset[0, \infty)$. It is well known [15], p.11, that there exists a constant $C>0$ such that

$$
K_{\phi_{\tau}}(f ; t) \leq C \omega_{\phi_{\tau}}(f ; t)
$$

Theorem 1 Iff $\in C_{B}[0, \infty)$, then

$$
\left\|V_{n}^{\left\langle\frac{1}{n}, \tau\right\rangle} f\right\| \leq\|f\| .
$$

Proof By the definition of the modified Stancu-Baskakov operators (2.1) and using Lemma 4 we have

$$
\left|V_{n}^{\left\langle\frac{1}{n}, \tau\right\rangle}(f ; x)\right| \leq \sum_{k=0}^{n} p_{n, k}^{\left\langle\frac{1}{n}, \tau\right\rangle}(x)\left|\left(f \circ \tau^{-1}\right)\left(\frac{k}{n}\right)\right| \leq\left\|f \circ \tau^{-1}\right\| V_{n}^{\left\langle\frac{1}{n}, \tau\right\rangle}(1 ; x)=\|f\|
$$

for every $x \in[0, \infty)$. Hence the required result is immediate.

Theorem 2 Let $f \in C_{B}[0, \infty)$. Then, for $n \geq 3$, there exists a constant $C>0$ such that

$$
\left|V_{n}^{\left\langle\frac{1}{n}, \tau\right\rangle}(f ; x)-f(x)\right| \leq C\left\{\omega_{2}\left(f ; \frac{\phi_{\tau}(x)}{\sqrt{n-2}}\right)+\frac{\phi_{\tau}^{2}(x)}{n-2}\|f\|\right\}+\omega\left(f \circ \tau^{-1} ;\left(\frac{\tau(x)}{n-1}\right)\right),
$$

on each compact subset of $[0, \infty)$. 
Proof Let $U$ be a compact subset of $[0, \infty)$. For each $x \in U$, first we define an auxiliary operator as

$$
V_{n}^{*\left(\frac{1}{n}, \tau\right\rangle}(f ; x)=V_{n}^{\left\langle\frac{1}{n}, \tau\right\rangle}(f ; x)-f \circ \tau^{-1}\left(\frac{n \tau(x)}{n-1}\right)+f(x) .
$$

Now, using Lemma 4, we have

$$
V_{n}^{*\left(\frac{1}{n}, \tau\right\rangle}(1 ; x)=1 \quad \text { and } \quad V_{n}^{*\left\langle\frac{1}{n}, \tau\right\rangle}(\tau(t) ; x)=\tau(x) \quad \text { hence } V_{n}^{*\left\langle\frac{1}{n}, \tau\right\rangle}(\tau(t)-\tau(x) ; x)=0 .
$$

Let $g \in W^{2}, x \in U$ and $t \in[0, \infty)$. Then by Taylor's expansion, and using results in [16], p.32, we get

$$
\begin{aligned}
g(t)= & \left(g \circ \tau^{-1}\right)(\tau(t)) \\
= & \left(g \circ \tau^{-1}\right)(\tau(x))+\left(g \circ \tau^{-1}\right)^{\prime}(\tau(x))(\tau(t)-\tau(x))+\int_{\tau(x)}^{\tau(t)}(\tau(t)-u)\left(g \circ \tau^{-1}\right)^{\prime \prime}(u) d u \\
= & g(x)+\left(g \circ \tau^{-1}\right)^{\prime}(\tau(x))(\tau(t)-\tau(x))+\int_{\tau(x)}^{\tau(t)}(\tau(t)-u) \frac{g^{\prime \prime}\left(\tau^{-1}(u)\right)}{\left[\tau^{\prime}\left(\tau^{-1}(u)\right)\right]^{2}} d u \\
& -\int_{\tau(x)}^{\tau(t)}(\tau(t)-u) \frac{g^{\prime}\left(\tau^{-1}(u)\right) \tau^{\prime \prime}\left(\tau^{-1}(u)\right)}{\left[\tau^{\prime}\left(\tau^{-1}(u)\right)\right]^{3}} d u .
\end{aligned}
$$

Now, applying the operator $V_{n}^{*\left\langle\frac{1}{n}, \tau\right\rangle}(\cdot ; x)$ to both sides of the above equality, we get

$$
\begin{aligned}
V_{n}^{*\left(\frac{1}{n}, \tau\right\rangle}(g ; x)-g(x) & \\
= & \left(g \circ \tau^{-1}\right)^{\prime}(\tau(x)) V_{n}^{*\left(\frac{1}{n}, \tau\right\rangle}((\tau(t)-\tau(x)) ; x) \\
& +V_{n}^{*\left\langle\frac{1}{n}, \tau\right\rangle}\left(\int_{\tau(x)}^{\tau(t)}(\tau(t)-u) \frac{g^{\prime \prime}\left(\tau^{-1}(u)\right)}{\left[\tau^{\prime}\left(\tau^{-1}(u)\right)\right]^{2}} d u ; x\right) \\
& -V_{n}^{*\left(\frac{1}{n}, \tau\right\rangle}\left(\int_{\tau(x)}^{\tau(t)}(\tau(t)-u) \frac{g^{\prime}\left(\tau^{-1}(u)\right) \tau^{\prime \prime}\left(\tau^{-1}(u)\right)}{\left[\tau^{\prime}\left(\tau^{-1}(u)\right)\right]^{3}} d u ; x\right) \\
= & V_{n}^{\left\langle\frac{1}{n}, \tau\right\rangle}\left(\int_{\tau(x)}^{\tau(t)}(\tau(t)-u) \frac{g^{\prime \prime}\left(\tau^{-1}(u)\right)}{\left[\tau^{\prime}\left(\tau^{-1}(u)\right)\right]^{2}} d u ; x\right) \\
& -\int_{\tau(x)}^{\frac{n \tau(x)}{n-1}}\left(\frac{n \tau(x)}{n-1}-u\right) \frac{g^{\prime \prime}\left(\tau^{-1}(u)\right)}{\left[\tau^{\prime}\left(\tau^{-1}(u)\right)\right]^{2}} d u \\
& -V_{n}^{\left\langle\frac{1}{n}, \tau\right\rangle}\left(\int_{\tau(x)}^{\tau(t)}(\tau(t)-u) \frac{g^{\prime}\left(\tau^{-1}(u)\right) \tau^{\prime \prime}\left(\tau^{-1}(u)\right)}{\left[\tau^{\prime}\left(\tau^{-1}(u)\right)\right]^{3}} d u ; x\right) \\
& +\int_{\tau(x)}^{\frac{n \tau(x)}{n-1}}\left(\frac{n \tau(x)}{n-1}-u\right) \frac{g^{\prime}\left(\tau^{-1}(u)\right) \tau^{\prime \prime}\left(\tau^{-1}(u)\right)}{\left[\tau^{\prime}\left(\tau^{-1}(u)\right)\right]^{3}} d u .
\end{aligned}
$$

Again, for each $x \in U$, we have

$$
\begin{aligned}
& \left|V_{n}^{*\left(\frac{1}{n}, \tau\right\rangle}(g ; x)-g(x)\right| \\
& \quad \leq \frac{1}{2} \frac{\left\|g^{\prime \prime}\right\|}{a^{2}} V_{n}^{\left\langle\frac{1}{n}, \tau\right\rangle}\left((\tau(t)-\tau(x))^{2} ; x\right)+\frac{1}{2} \frac{\left\|g^{\prime \prime}\right\|}{a^{2}}\left(\frac{n \tau(x)}{n-1}-\tau(x)\right)^{2}
\end{aligned}
$$




$$
\begin{aligned}
& +\frac{1}{2} \frac{\left\|g^{\prime}\right\|\left\|\tau^{\prime \prime}\right\|}{a^{3}} V_{n}^{\left\langle\frac{1}{n}, \tau\right\rangle}\left((\tau(t)-\tau(x))^{2} ; x\right)+\frac{1}{2} \frac{\left\|g^{\prime}\right\|\left\|\tau^{\prime \prime}\right\|}{a^{3}}\left(\frac{n \tau(x)}{n-1}-\tau(x)\right)^{2} \\
= & \frac{1}{2}\left(\frac{\left\|g^{\prime \prime}\right\|}{a^{2}}+\frac{\left\|g^{\prime}\right\|\left\|\tau^{\prime \prime}\right\|}{a^{3}}\right)\left[V_{n}^{\left\langle\frac{1}{n}, \tau\right\rangle}\left((\tau(t)-\tau(x))^{2} ; x\right)+\left(\frac{\tau(x)}{n-1}\right)^{2}\right] .
\end{aligned}
$$

Now, using the definition of the auxiliary operators, Theorem 1 and inequality (2.7), for each $x \in U$ we have

$$
\begin{aligned}
& \left|V_{n}^{\left\langle\frac{1}{n}, \tau\right\rangle}(f ; x)-f(x)\right| \\
& \leq\left|V_{n}^{*\left\langle\frac{1}{n}, \tau\right\rangle}(f-g ; x)\right|+\left|V_{n}^{*\left(\frac{1}{n}, \tau\right\rangle}(g ; x)-g(x)\right| \\
& \quad+|g(x)-f(x)|+\left|f \circ \tau^{-1}\left(\frac{n \tau(x)}{n-1}\right)-f \circ \tau^{-1}(\tau(x))\right| \\
& \leq 4\|f-g\|+\frac{1}{2}\left(\frac{\left\|g^{\prime \prime}\right\|}{a^{2}}+\frac{\left\|g^{\prime}\right\|\left\|\tau^{\prime \prime}\right\|}{a^{3}}\right)\left[V_{n}^{\left\langle\frac{1}{n}, \tau\right\rangle}\left((\tau(t)-\tau(x))^{2} ; x\right)+\left(\frac{\tau(x)}{n-1}\right)^{2}\right] \\
& \quad+\omega\left(f \circ \tau^{-1} ;\left(\frac{\tau(x)}{n-1}\right)\right) .
\end{aligned}
$$

Let $C=\max \left(4, \frac{4}{a^{2}}, \frac{4}{a^{3}}\left\|\tau^{\prime \prime}\right\|\right)$, we get

$$
\left|V_{n}^{\left\langle\frac{1}{n}, \tau\right\rangle}(f ; x)-f(x)\right| \leq C\left(\|f-g\|+\|g\|_{W^{2}} \frac{\phi_{\tau}^{2}(x)}{n-2}\right)+\omega\left(f \circ \tau^{-1} ;\left(\frac{\tau(x)}{n-1}\right)\right) .
$$

Taking the infimum on the right side of the above inequality over all $g \in W^{2}$ and for all $x \in U$, we have

$$
\left|V_{n}^{\left\langle\frac{1}{n}, \tau\right\rangle}(f ; x)-f(x)\right| \leq C K\left(f ; \frac{\phi_{\tau}^{2}(x)}{n-2}\right)+\omega\left(f \circ \tau^{-1} ;\left(\frac{\tau(x)}{n-1}\right)\right),
$$

using equation (2.2), we get the required result.

Theorem 3 Let $f \in C_{B}[0, \infty)$. Then for every $x \in[0, \infty)$, and $n \geq 3$ we have

$$
\left|V_{n}^{\left\langle\frac{1}{n}, \tau\right\rangle}(f ; x)-f(x)\right| \leq C \omega_{\phi_{\tau}}\left(f ; \frac{\sqrt{6} c(x)}{a \sqrt{(n-2)}}\right) .
$$

Proof For any $g \in W_{\phi_{\tau}}[0, \infty)$, by Taylor's expansion, we have

$$
g(t)=\left(g \circ \tau^{-1}\right)(\tau(t))=\left(g \circ \tau^{-1}\right)(\tau(x))+\int_{\tau(x)}^{\tau(t)}\left(g \circ \tau^{-1}\right)^{\prime}(u) d u .
$$

Applying the operator $V_{n}^{\left\langle\frac{1}{n}, \tau\right\rangle}(\cdot ; x)$ on both sides of the above equality, we get

$$
\left|V_{n}^{\left\langle\frac{1}{n}, \tau\right\rangle}(g ; x)-g(x)\right|=\left|V_{n}^{\left\langle\frac{1}{n}, \tau\right\rangle}\left(\int_{\tau(x)}^{\tau(t)}\left(g \circ \tau^{-1}\right)^{\prime}(u) d u\right)\right| .
$$


From [16], we have

$$
\begin{aligned}
\left|\int_{\tau(x)}^{\tau(t)}\left(g \circ \tau^{-1}\right)^{\prime}(u) d u\right| & =\left|\int_{x}^{t} \frac{g^{\prime}(y)}{\tau^{\prime}(y)} \tau^{\prime}(y) d y\right|=\left|\int_{x}^{t} \frac{\phi_{\tau}(y)}{\phi_{\tau}(y)} \frac{g^{\prime}(y)}{\tau^{\prime}(y)} \tau^{\prime}(y) d y\right| \\
& \leq \frac{\left\|\phi_{\tau} g^{\prime}\right\|}{a}\left|\int_{x}^{t} \frac{\tau^{\prime}(y)}{\phi_{\tau}(y)} d y\right|
\end{aligned}
$$

and

$$
\begin{aligned}
\left|\int_{x}^{t} \frac{\tau^{\prime}(y)}{\phi_{\tau}(y)} d y\right| & \leq\left|\int_{x}^{t}\left(\frac{1}{\sqrt{\tau(y)}}+\frac{1}{\sqrt{1+\tau(y)}}\right) \tau^{\prime}(y) d y\right| \\
& \leq\left|\int_{x}^{t} \frac{1}{\sqrt{\tau(y)}} \tau^{\prime}(y) d y\right|+\left|\int_{x}^{t} \frac{1}{\sqrt{1+\tau(y)}} \tau^{\prime}(y) d y\right| \\
& =2\{|\sqrt{\tau(t)}-\sqrt{\tau(x)}|+|\sqrt{1+\tau(t)}-\sqrt{1+\tau(x)}|\} \\
& <2|\tau(t)-\tau(x)|\left(\frac{1}{\sqrt{\tau(x)}}+\frac{1}{\sqrt{1+\tau(x)}}\right) \\
& =\frac{2|\tau(t)-\tau(x)|}{\sqrt{\tau(x)(1+\tau(x))}}(\sqrt{1+\tau(x)}+\sqrt{\tau(x)}) \\
& =\frac{2|\tau(t)-\tau(x)|}{\sqrt{\tau(x)(1+\tau(x))}} c(x) \\
& =\frac{2 c(x)|\tau(t)-\tau(x)|}{\phi_{\tau}(x)} .
\end{aligned}
$$

Now, from equations (2.12)-(2.13) and using the Cauchy-Schwarz inequality, we obtain

$$
\begin{aligned}
\left|V_{n}^{\left\langle\frac{1}{n}, \tau\right\rangle}(g ; x)-g(x)\right| & \leq \frac{2 c(x)\left\|\phi_{\tau} g^{\prime}\right\|}{a \phi_{\tau}(x)} V_{n}^{\left\langle\frac{1}{n}, \tau\right\rangle}(|\tau(t)-\tau(x)| ; x) \\
& \leq \frac{2 c(x)\left\|\phi_{\tau} g^{\prime}\right\|}{a \phi_{\tau}(x)} V_{n}^{\left\langle\frac{1}{n}, \tau\right\rangle}\left((\tau(t)-\tau(x))^{2} ; x\right)^{\frac{1}{2}} \\
& =\frac{2 c(x)\left\|\phi_{\tau} g^{\prime}\right\|}{a \phi_{\tau}(x)}\left[\frac{2 n \phi_{\tau}^{2}(x)+(2 \tau(x)-1) \tau(x)}{(n-1)(n-2)}\right]^{\frac{1}{2}} .
\end{aligned}
$$

Thus, for $f \in C_{B}[0, \infty)$ and any $g \in W_{\phi_{\tau}}[0, \infty)$, we have

$$
\begin{aligned}
\left|V_{n}^{\left\langle\frac{1}{n}, \tau\right\rangle}(f ; x)-f(x)\right| & \leq\left|V_{n}^{\left\langle\frac{1}{n}, \tau\right\rangle}(f-g ; x)\right|+|f(x)-g(x)|+\left|V_{n}^{\left\langle\frac{1}{n}, \tau\right\rangle}(g ; x)-g(x)\right| \\
& \leq 2\|f-g\|+\frac{2 c(x)\left\|\phi_{\tau} g^{\prime}\right\|}{a \phi_{\tau}(x)}\left[\frac{2 n \phi_{\tau}^{2}(x)+(2 \tau(x)-1) \tau(x)}{(n-1)(n-2)}\right]^{\frac{1}{2}} \\
& =\frac{2 c(x)\left\|\phi_{\tau} g^{\prime}\right\|}{a}\left[\frac{2(n+1)}{(n-1)(n-2)}\right]^{\frac{1}{2}}+2\|f-g\| \\
& \leq 2\left\{\|f-g\|+\frac{\sqrt{6} c(x)}{a \sqrt{(n-2)}}\left\|\phi_{\tau} g^{\prime}\right\|\right\} .
\end{aligned}
$$


Taking the infimum on the right side of the above inequality over all $g \in W_{\phi_{\tau}}[0, \infty)$, we get

$$
\left|V_{n}^{\left\langle\frac{1}{n}, \tau\right\rangle}(f ; x)-f(x)\right| \leq 2 K_{\phi_{\tau}}\left(f ; \frac{\sqrt{6} c(x)}{a \sqrt{(n-2)}}\right) .
$$

Finally, using equation (2.3), the theorem is immediate.

Theorem 4 For any $f \in C^{2}[0, \infty)$ and $x \in[0, \infty)$, the following inequality hold:

$$
\begin{aligned}
& \left|V_{n}^{\left\langle\frac{1}{n}, \tau\right\rangle}(f ; x)-f(x)-\frac{f^{\prime}(x)}{\tau^{\prime}(x)} \mu_{n, 1}^{\tau}(x)-\frac{1}{2}\left[\frac{f^{\prime \prime}(x)}{\left(\tau^{\prime}(x)\right)^{2}}-f^{\prime}(x) \frac{\tau^{\prime \prime}(x)}{\left(\tau^{\prime}(x)\right)^{3}}\right] \mu_{n, 2}^{\tau}(x)\right| \\
& \quad \leq\left(\mu_{n, 2}^{\tau}(x)\right)^{\frac{1}{2}}\left[\left\|\left(f \circ \tau^{-1}\right)^{\prime \prime}-g\right\|\left(\mu_{n, 2}^{\tau}(x)\right)^{\frac{1}{2}}+\frac{2\left\|\phi_{\tau} g^{\prime}\right\|}{a} \phi_{\tau}^{-1}(x)\left(\mu_{n, 4}^{\tau}(x)\right)^{1 / 2}\right] .
\end{aligned}
$$

Proof Let $f \in C^{2}[0, \infty)$ and $x, t \in[0, \infty)$. Then by Taylor's expansion, we have

$$
\begin{aligned}
f(t) & =\left(f \circ \tau^{-1}\right)(\tau(t)) \\
& =\left(f \circ \tau^{-1}\right)(\tau(x))+\left(f \circ \tau^{-1}\right)^{\prime}(\tau(x))(\tau(t)-\tau(x))+\int_{\tau(x)}^{\tau(t)}(\tau(t)-u)\left(f \circ \tau^{-1}\right)^{\prime \prime}(u) d u .
\end{aligned}
$$

Hence,

$$
\begin{aligned}
f(t) & -f(x)-\left(f \circ \tau^{-1}\right)^{\prime}(\tau(x))(\tau(t)-\tau(x))-\frac{1}{2}\left(f \circ \tau^{-1}\right)^{\prime \prime}(\tau(x))(\tau(t)-\tau(x))^{2} \\
= & \int_{\tau(x)}^{\tau(t)}(\tau(t)-u)\left(f \circ \tau^{-1}\right)^{\prime \prime}(u) d u-\int_{\tau(x)}^{\tau(t)}(\tau(t)-u)\left(f \circ \tau^{-1}\right)^{\prime \prime}(\tau(x)) d u \\
= & \int_{\tau(x)}^{\tau(t)}(\tau(t)-u)\left[\left(f \circ \tau^{-1}\right)^{\prime \prime}(u)-\left(f \circ \tau^{-1}\right)^{\prime \prime}(\tau(x))\right] d u .
\end{aligned}
$$

Applying $V_{n}^{\left\langle\frac{1}{n}, \tau\right\rangle}$ to both sides of the above relation, we get

$$
\begin{gathered}
\left|V_{n}^{\left\langle\frac{1}{n}, \tau\right\rangle}(f ; x)-f(x)-\frac{f^{\prime}(x)}{\tau^{\prime}(x)} \mu_{n, 1}^{\tau}(x)-\frac{1}{2}\left[\frac{f^{\prime \prime}(x)}{\left(\tau^{\prime}(x)\right)^{2}}-f^{\prime}(x) \frac{\tau^{\prime \prime}(x)}{\left(\tau^{\prime}(x)\right)^{3}}\right] \mu_{n, 2}^{\tau}(x)\right| \\
\quad=\left|V_{n}^{\left\langle\frac{1}{n}, \tau\right\rangle}\left(\int_{\tau(x)}^{\tau(t)}(\tau(t)-u)\left[\left(f \circ \tau^{-1}\right)^{\prime \prime}(u)-\left(f \circ \tau^{-1}\right)^{\prime \prime}(\tau(x))\right] d u ; x\right)\right| \\
\quad \leq V_{n}^{\left\langle\frac{1}{n}, \tau\right\rangle}\left(\left|\int_{\tau(x)}^{\tau(t)}\right| \tau(t)-u||\left(f \circ \tau^{-1}\right)^{\prime \prime}(u)-\left(f \circ \tau^{-1}\right)^{\prime \prime}(\tau(x))|d u| ; x\right) .
\end{gathered}
$$

For $g \in W_{\phi_{\tau}[0, \infty)}$, we have

$$
\begin{aligned}
& \left|\int_{\tau(x)}^{\tau(t)}\right| \tau(t)-u||\left(f \circ \tau^{-1}\right)^{\prime \prime}(u)-\left(f \circ \tau^{-1}\right)^{\prime \prime}(\tau(x))|d u| \\
& \leq\left|\int_{\tau(x)}^{\tau(t)}\right| \tau(t)-u||\left(f \circ \tau^{-1}\right)^{\prime \prime}(u)-\left(g \circ \tau^{-1}\right)(u)|d u| \\
& \quad+\left|\int_{\tau(x)}^{\tau(t)}\right| \tau(t)-u||\left(g \circ \tau^{-1}\right)(u)-\left(g \circ \tau^{-1}\right)(\tau(x))|d u|
\end{aligned}
$$


Deshwal et al. Journal of Inequalities and Applications （2017) 2017:57

Page 10 of 11

$$
\begin{aligned}
& +\left|\int_{\tau(x)}^{\tau(t)}\right| \tau(t)-u||\left(g \circ \tau^{-1}\right)(\tau(x))-\left(f \circ \tau^{-1}\right)^{\prime \prime}(\tau(x))|d u| \\
= & \left|\int_{x}^{t}\right|\left(f \circ \tau^{-1}\right)^{\prime \prime}(\tau(y))-g(y)|| \tau(t)-\tau(y)\left|\tau^{\prime}(y) d y\right| \\
& +\left|\int_{x}^{t}\right| g(y)-g(x)|| \tau(t)-\tau(y)\left|\tau^{\prime}(y) d y\right| \\
& +\left|\int_{x}^{t}\right| g(x)-\left(f \circ \tau^{-1}\right)^{\prime \prime}(\tau(x))|| \tau(t)-\tau(y)\left|\tau^{\prime}(y) d y\right| \\
\leq & 2\left\|\left(f \circ \tau^{-1}\right)^{\prime \prime}-g\right\|\left|\int_{x}^{t}\right| \tau(t)-\tau(y)\left|\tau^{\prime}(y) d y\right| \\
& +\left|\int_{x}^{t}\right| \int_{x}^{y}\left|g^{\prime}(v)\right| d v|| \tau(t)-\tau(y)\left|\tau^{\prime}(y) d y\right| \\
\leq & \left\|\left(f \circ \tau^{-1}\right)^{\prime \prime}-g\right\|(\tau(t)-\tau(x))^{2}+\left\|\phi_{\tau} g^{\prime}\right\|\left|\int_{x}^{t}\right| \int_{x}^{y} \frac{d v}{\phi_{\tau}(v)}|| \tau(t)-\tau(y)\left|\tau^{\prime}(y) d y\right| .
\end{aligned}
$$

Using the inequality

$$
\frac{|y-v|}{v(1+v)} \leq \frac{|y-x|}{x(1+x)}, \quad x<v<y,
$$

we can write

$$
\frac{|\tau(y)-\tau(v)|}{\tau(v)(1+\tau(v))} \leq \frac{|\tau(y)-\tau(x)|}{\tau(x)(1+\tau(x))} .
$$

Therefore,

$$
\begin{aligned}
& \left|\int_{\tau(x)}^{\tau(t)}\right| \tau(t)-u \|\left(f \circ \tau^{-1}\right)^{\prime \prime}(u)-\left(f \circ \tau^{-1}\right)^{\prime \prime}(\tau(x))|d u| \\
& \quad \leq\left\|\left(f \circ \tau^{-1}\right)^{\prime \prime}-g\right\|(\tau(t)-\tau(x))^{2} \\
& \quad+\left\|\phi_{\tau} g^{\prime}\right\|\left|\int_{x}^{t}\right| \int_{x}^{y} \frac{|\tau(y)-\tau(x)|^{1 / 2}}{\tau^{\prime}(v) \phi_{\tau}(x)} \cdot \frac{\tau^{\prime}(v)}{|\tau(y)-\tau(v)|^{1 / 2}} d \nu|| \tau(t)-\tau(y)\left|\tau^{\prime}(y) d y\right| \\
& \leq\left\|\left(f \circ \tau^{-1}\right)^{\prime \prime}-g\right\|(\tau(t)-\tau(x))^{2} \\
& \quad+2 \frac{\left\|\phi_{\tau} g^{\prime}\right\|}{a} \phi_{\tau}^{-1}(x)\left|\int_{x}^{t}\right| \tau(y)-\tau(x)|| \tau(t)-\tau(y)\left|\tau^{\prime}(y) d y\right| \\
& \leq\left\|\left(f \circ \tau^{-1}\right)^{\prime \prime}-g\right\|(\tau(t)-\tau(x))^{2}+2 \frac{\left\|\phi_{\tau} g^{\prime}\right\|}{a} \phi_{\tau}^{-1}(x)\left|\int_{x}^{t}(\tau(t)-\tau(x))^{2} \tau^{\prime}(y) d y\right| \\
& \quad \leq\left\|\left(f \circ \tau^{-1}\right)^{\prime \prime}-g\right\|(\tau(t)-\tau(x))^{2}+2 \frac{\left\|\phi_{\tau} g^{\prime}\right\|}{a} \phi_{\tau}^{-1}(x)|\tau(t)-\tau(x)|^{3} .
\end{aligned}
$$

Now combining equations (2.16)-(2.17), applying Lemma 3 and the Cauchy-Schwarz inequality, we get

$$
\begin{aligned}
& \left|V_{n}^{\left\langle\frac{1}{n}, \tau\right\rangle}(f ; x)-f(x)-\frac{f^{\prime}(x)}{\tau^{\prime}(x)} \mu_{n, 1}^{\tau}(x)-\frac{1}{2}\left[\frac{f^{\prime \prime}(x)}{\left(\tau^{\prime}(x)\right)^{2}}-f^{\prime}(x) \frac{\tau^{\prime \prime}(x)}{\left(\tau^{\prime}(x)\right)^{3}}\right] \mu_{n, 2}^{\tau}(x)\right| \\
& \quad \leq\left\|\left(f \circ \tau^{-1}\right)^{\prime \prime}-g\right\| V_{n}^{\left\langle\frac{1}{n}, \tau\right\rangle}\left((\tau(t)-\tau(x))^{2} ; x\right)+2 \frac{\left\|\phi_{\tau} g^{\prime}\right\|}{a} \phi_{\tau}^{-1}(x) V_{n}^{\left\langle\frac{1}{n}, \tau\right\rangle}\left(|\tau(t)-\tau(x)|^{3} ; x\right)
\end{aligned}
$$




$$
\begin{aligned}
\leq & \left\|\left(f \circ \tau^{-1}\right)^{\prime \prime}-g\right\| V_{n}^{\left\langle\frac{1}{n}, \tau\right\rangle}\left((\tau(t)-\tau(x))^{2} ; x\right) \\
& +\frac{2\left\|\phi_{\tau} g^{\prime}\right\|}{a} \phi_{\tau}^{-1}(x)\left[V_{n}^{\left\langle\frac{1}{n}, \tau\right\rangle}\left((\tau(t)-\tau(x))^{2} ; x\right)\right]^{1 / 2}\left[V_{n}^{\left\langle\frac{1}{n}, \tau\right\rangle}\left((\tau(t)-\tau(x))^{4} ; x\right)\right]^{1 / 2} \\
= & \left\|\left(f \circ \tau^{-1}\right)^{\prime \prime}-g\right\| \mu_{n, 2}^{\tau}(x)+\frac{2\left\|\phi_{\tau} g^{\prime}\right\|}{a} \phi_{\tau}^{-1}(x)\left(\mu_{n, 2}^{\tau}(x)\right)^{1 / 2}\left(\mu_{n, 4}^{\tau}(x)\right)^{1 / 2} \\
= & \left(\mu_{n, 2}^{\tau}(x)\right)^{\frac{1}{2}}\left[\left\|\left(f \circ \tau^{-1}\right)^{\prime \prime}-g\right\|\left(\mu_{n, 2}^{\tau}(x)\right)^{\frac{1}{2}}+\frac{2\left\|\phi_{\tau} g^{\prime}\right\|}{a} \phi_{\tau}^{-1}(x)\left(\mu_{n, 4}^{\tau}(x)\right)^{1 / 2}\right] .
\end{aligned}
$$

\section{This completes the proof of the theorem.}

\section{Competing interests}

The authors declare that they have no competing interests.

\section{Authors' contributions}

All authors equally contributed to this work. All authors read and approved the final manuscript.

\section{Author details}

${ }^{1}$ Indian Institute of Techology Roorkee, Roorkee, 247667, India. ${ }^{2}$ Department of Economics, Faculty of Economics, Administrative and Social Sciences, Hasan Kalyoncu University, Gaziantep, 27410, Turkey.

\section{Acknowledgements}

The first author is thankful to The Ministry of Human Resource and Development, India, for the financial support to carry out the above work.

Received: 15 December 2016 Accepted: 20 February 2017 Published online: 06 March 2017

\section{References}

1. Eggenberger, F, Polya, G: Über die statistik verkerter vorgänge. Z. Angew. Math. Mech. 1, $279-289$ (1923)

2. Johnson, NL, Kotz, S: Discrete Distributions. Houghton Mifflin, Boston (1969)

3. Stancu, DD: Two classes of positive linear operators. An. Univ. Vest. Timiş., Ser. Mat.-Inform. 8, $213-220$ (1970)

4. Baskakov, VA: A sequence of linear positive operators in the space of continuous functions. Dokl. Akad. Nauk SSSR 113, 249-251 (1957)

5. Razi, Q: Approximation of a function by Kantrovich type operators. Mat. Vesn. 41, 183-192 (1989)

6. Deo, N, Dhamija, M, Miclăus, D: Stancu-Kantorovich operators based on inverse Pólya-Eggenberger distributions. Appl. Math. Comput. 273, 281-289 (2016)

7. Acar, T: Asymptotic formulas for generalized Szasz-Mirakyan operators. Appl. Math. Comput. 263, 233-239 (2015)

8. Acar, T: (p,q)-Generalization of Szasz-Mirakan operators. Math. Methods Appl. Sci. 39(10), 2685-2695 (2016)

9. Acar, T: Quantitative $q$-Voronovskaya and $q$-Grüss-Voronovskaya-type results for $q$-Szász operators. Georgian Math. J. 23(4), 459-468 (2016)

10. Stancu, DD: Approximation of functions by a new class of linear polynomial operators. Rev. Roum. Math. Pures Appl. 13, 1173-1194 (1968)

11. King, JP: Positive linear operators which preserve $x^{2}$. Acta Math. Hung. 99, 203-208 (2003)

12. Gonska, H, Piţul, P, Raşa, I: General King-type operators. Results Math. 53, 279-286 (2009)

13. Peetre, J: Theory of Interpolation of Normed Spaces, 86 pp. Notas de Matematica, vol. 39. Rio de Janeiro (1963)

14. Butzer, PL, Berens, H: Semi-Groups of Operators and Approximation. Springer, Berlin (1967)

15. Ditzian, Z, Totik, V: Moduli of Smoothness. Springer, New York (1987)

16. Acar, T, Aral, A, Raşa, I: Modified Bernstein-Durrmeyer operators. Gen. Math. 22(1), 27-41 (2014) 